\title{
Effects of overmodulation in fixation-free rehalogenating bleached holograms
}

\author{
Cristian Neipp, Inmaculada Pascual, and Augusto Beléndez
}

\begin{abstract}
The mechanism for hologram formation by fixation-free rehalogenating bleaching has been treated by several authors. Experiments carried out with Kodak and Agfa photographic plates have led to the conclusion that the transfer of material from the exposed to the unexposed zones is the main mechanism that drives the process. We show that when BB-640 emulsion is used in the recording of holograms by means of fixation-free rehalogenating bleaching, the mechanism for the transfer of material from exposed to unexposed zones is efficient enough to create high refractive-index modulations. We also comment on the overmodulation effects, which result in a decrease in diffraction efficiency. (C) 2001 Optical Society of America

OCIS codes: $\quad 090.0090,090.2900,090.7330$.
\end{abstract}

\section{Introduction}

Volume phase holograms are desirable for use as holographic optical elements owing to their high diffraction efficiency. ${ }^{1}$ One of the commonest holographic materials is photographic emulsion. Volume holograms recorded in silver halide emulsions are commonly bleached to yield phase holograms to increase diffraction efficiency. In particular, reversal ${ }^{2,3}$ and fixation-free rehalogenating methods ${ }^{4,5}$ have been used in recent decades, mainly with Kodak and Agfa plates. The chemical processes involved in rehalogenating bleaching without a fixation step have been the subject of much controversy. In these processes, after development, the emulsion is rehalogenated and the resultant change in thickness of the emulsion produced by these rehalogenating baths is small. In addition, it is assumed that the average refractive index of the emulsion does not change appreciably as a result of processing. ${ }^{6}$ The mechanism for hologram formation by fixation-free rehalogenating bleaching was studied by Hariharan.7,8 It is assumed that diffusion of material from the exposed to

C. Neipp and A. Beléndez (augusto@disc.ua.es) are with the Departamento de Física, Ingeniería de Sistemas y Teoría de la Señal, Universidad de Alicante, Apartado 99, E-03080 Alicante, Spain. I. Pascual is with the Departamento Interuniversitario de Óptica, Universidad de Alicante, Apartado 99, E-03080 Alicante, Spain.

Received 21 December 2000; revised manuscript received 29 March 2001.

0003-6935/01/203402-07\$15.00/0

(C) 2001 Optical Society of America the unexposed zones occurs during the bleach bath. The metallic silver grains are converted back into silver halide grains during the bleach bath by an oxidation process. After this oxidation, some silver ions go into solution, some of them are deposited in situ in the exposed zones, and others diffuse away to the unexposed zones. The refractive-index modulation between the exposed and the nonexposed zones is produced by differences in sizes of the silver halide grains.

BB-640 ultrafine-grained red-sensitive emulsions from Holographic Recording Technologies were used in our experiments. There are some differences between BB-640 and Agfa 8E75 HD plates, ${ }^{9-12}$ so the effects of the transfer of material may be different for the two plates. For instance, the gelatin of BB-640 plates is hardened to a higher degree than that of Agfa 8E75 HD plates, and the silver halide grains of BB-640 plates are smaller. In this paper we examine in detail the mechanism for hologram formation in the bleaching process on BB-640 plates, using the fixation-free rehalogenating method. We demonstrate that high refractive-index modulations are obtained, which in fact produce a decrease in diffraction efficiency at certain exposures. We also compare the results obtained for bleached holograms recorded on BB-640 plates with those obtained for silver halidesensitized gelatin (SHSG) holograms recorded on the same emulsion.

\section{Experimental Procedure}

Figure 1 shows the experimental setup used in the experiments. Unslanted holographic transmission 


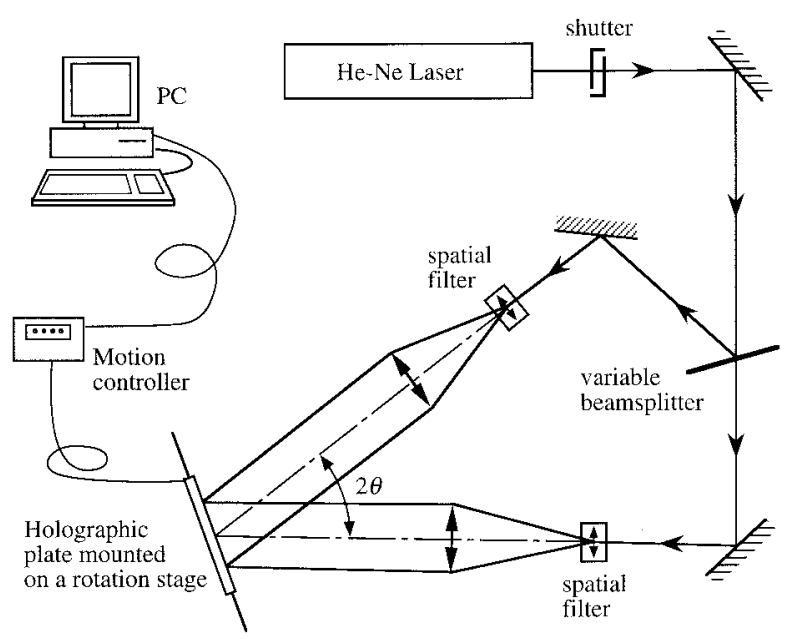

Fig. 1. Experimental setup.

gratings were recorded by use of two collimated beams from a $15-\mathrm{mW} \mathrm{He}-\mathrm{Ne}$ laser $(633 \mathrm{~nm})$ with the polarization vector perpendicular to the plane of incidence. The two beams, of equal intensity, impinged upon the emulsion, forming an angle (in air) of $45^{\circ}$. With the geometry described, the spatial frequency of the gratings was calculated as 1200 lines/ mm. A black self-adhesive polyvinyl chloride masking tape was attached to the back of each holographic plate to prevent unwanted reflections from occurring at the glass-air interface of the plates during recording of the holograms. ${ }^{13}$ Holographic plates were mounted upon a motorized rotation stage, which was controlled electronically with a dc point-to-point motion controller connected to a personal computer by an IEEE-488 interface. The rotating device has a resolution of $0.001^{\circ}$.

In previous experiments ${ }^{10}$ we found that the gelatin of BB-640 plates is hardened to a high degree, and so the products of the developer do not easily penetrate into the emulsion. To soften the gelatin, we hypersensitized the plates by immersing them in a solution of distilled water with a sodium sulfite concentration of $1 \%$ and a urea concentration of $5 \%$ (by weight) for $10 \mathrm{~min}$ at $20^{\circ} \mathrm{C}$. After they were rinsed in running water for $1 \mathrm{~min}$ at $20^{\circ} \mathrm{C}$ the plates were dried for $24 \mathrm{~h}$ at $20^{\circ} \mathrm{C}$ and $60 \%$ relative humidity. The urea in this solution softened the gelatin, and the sensitivity of the emulsion was also increased.

After exposure, the plates underwent the scheduling procedure shown in Table 1, so two series of holograms were finally obtained: rehalogenated bleached transmission holograms and SHSG holograms. ${ }^{11}$ Details of the developer and bleach formulas are listed in Table 2. The results obtained for bleached holograms can be compared with those obtained with other procedures. The AAC developer, an ascorbic acid-based developer (which is nontanning) was used to minimize the hardening produced by the oxidation products of tanning developers during the development step. In the SHSG procedure we used a nonhardener fixer (F-24) to prevent differential hardening during the fixation bath. To increase the refractive-index modulation, we dehydrated the SHSG holograms in subsequent baths of isopropanol and distilled water at different concentrations. Finally, the plates were dried in a desiccator at a low relative humidity.

The diffraction efficiency $\eta$ of the recorded phase holograms was calculated as the ratio of the intensity of the diffracted beam to that of the incident collimated probe beam of a He-Ne laser. To take into account Fresnel losses and absorption caused by the glass substrate, we corrected this expression by multiplying it by an appropriate factor. We calculated the losses by using Fresnel equations for $s$-polarized light to take into account the reflections that take place in the different interfaces of the hologram. The efficiency of the zero order or of transmission $\tau$ was similarly calculated as the ratio of the directly transmitted beam intensity to the incident power and was corrected by the same factor.

\section{Preliminary Considerations}

To determine the values of the refractive-index modulation created inside the hologram recorded on BB640 plates, we used the Kogelnik coupled-wave theory. ${ }^{14}$ When losses occur in the holographic medium, the analytical expression for the diffraction

Table 1. Processing Schedule

Fixation-Free Bleached Holograms

\begin{tabular}{|c|c|c|c|}
\hline \multicolumn{2}{|l|}{ Fixation-Free Bleached Holograms } & \multicolumn{2}{|l|}{ Silver Halide-Sensitized Gelatin } \\
\hline 1. Develop in AAC $\left(20^{\circ} \mathrm{C}\right)$ & $5 \mathrm{~min}$ & 1. Develop in AAC $\left(20^{\circ} \mathrm{C}\right)$ & $5 \mathrm{~min}$ \\
\hline 2. Rinse in running water & $1 \mathrm{~min}$ & 2. Rinse in running water & $1 \mathrm{~min}$ \\
\hline 3. Bleach for 1 min after the plate has cleared & & 3. Bleach for 1 min after the plate has cleared & \\
\hline 4. Rinse in running water & $5 \min$ & 4. Rinse in running water & $5 \mathrm{~min}$ \\
\hline 5. Dry at room temperature & $24 \mathrm{~h}$ & 5. Dry at room temperature & $24 \mathrm{~h}$ \\
\hline & & 6. Rinse in running water & $10 \mathrm{~min}$ \\
\hline & & 7. Fix in nonhardener fixer (F-24) & $7 \mathrm{~min}$ \\
\hline & & 8. Dehydrate in $50 \%$ isopropanol & $3 \mathrm{~min}$ \\
\hline & & 9. Dehydrate in $90 \%$ isopropanol & $3 \mathrm{~min}$ \\
\hline & & 10. Dehydrate in $100 \%$ isopropanol & $3 \mathrm{~min}$ \\
\hline & & $\begin{array}{l}\text { 11. Dry in desiccator at } 20^{\circ} \mathrm{C} \text { and relative } \\
\text { humidity }<20 \%\end{array}$ & $24 \mathrm{~h}$ \\
\hline
\end{tabular}


Table 2. Developer and Bleach Formulas

\begin{tabular}{ll}
\hline \multicolumn{1}{c}{ Component } & Amount \\
\hline AAC Developer & \\
Solution A & \\
Ascorbic acid & $18 \mathrm{~g}$ \\
Distilled water & $0.25 \mathrm{~L}$ \\
Solution B & $60 \mathrm{~g}$ \\
$\quad$ Sodium carbonate (anhydrous) & $0.75 \mathrm{~L}$ \\
$\quad$ Distilled water & \\
Bleach formula (modified version of the & \\
Kodak bleach bath type R-10) & \\
Solution A & $20 \mathrm{~g}$ \\
$\quad$ Potassium dichromate & $15 \mathrm{~mL}$ \\
Sulfuric acid & $1 \mathrm{~L}$ \\
Distilled water & \\
Solution B & $100 \mathrm{~g}$ \\
Potassium bromide & $1 \mathrm{~L}$ \\
Distilled water &
\end{tabular}

${ }^{a}$ Just before use, mix A with B.

${ }^{b}$ Just before use, mix 1 part of $\mathrm{A}$ with 10 parts of distilled water and add 8 parts of $B$.

efficiency for pure phase thick transmission holographic gratings is obtained as follows,

$$
\eta=\exp \left(-\alpha d / \cos \theta^{\prime}\right) \frac{\sin ^{2}\left(v^{2}+\xi^{2}\right)^{1 / 2}}{1+\left(\xi^{2} / \nu^{2}\right)}
$$

where, for nonslanted gratings, $v$ and $\xi$ take the following values:

$$
\begin{aligned}
& v=\frac{\pi \Delta n d}{\lambda \cos \theta^{\prime}}, \\
& \xi=\frac{\pi d}{\Lambda \cos \theta^{\prime}}\left(\left|\sin \theta^{\prime}\right|-\frac{\lambda}{2 n \Lambda}\right) .
\end{aligned}
$$

For lossy-transmission pure phase gratings, the efficiency of the transmitted beam, $\tau$, can easily be obtained as follows ${ }^{15}$ :

$$
\tau=\exp \left(-\alpha d / \cos \theta^{\prime}\right)\left[1-\frac{\sin ^{2}\left(\nu^{2}+\xi^{2}\right)^{1 / 2}}{1+\left(\xi^{2} / \nu^{2}\right)}\right] .
$$

From Eqs. (1) and (4) it is easy to see that for lossytransmission pure phase gratings $\xi$ and $\tau$ verify the following equation:

$$
\eta+\tau=\exp \left(-\alpha d / \cos \theta^{\prime}\right)
$$

then the replay angle for maximum diffraction efficiency coincides with the replay angle for minimum transmission. ${ }^{15}$ In Eqs. (1)-(5) $\alpha$ takes into account the absorption (and also the scattering; we have no means of differentiating between the two at this point), $d$ is the thickness, $n$ is the mean refractive index, $\Delta n$ is the refractive-index modulation, $\Lambda$ is the grating period, $\lambda$ is the wavelength of reconstruction in air, and $\theta^{\prime}$ is the angle of reconstruction in the medium, which is related to the angle of reconstruction in air, $\theta$, by Snell's law.

For maximum diffraction efficiency at the Bragg angle $(\xi=0), v$ must take the value $\pi / 2$. For the gratings analyzed in this paper the Bragg angle in air is $\sim 22.5^{\circ}$ because unslanted diffraction gratings with an interbeam angle of $45^{\circ}$ were recorded. With Eq. (2) the value of the product $\Delta n d$ that yields a value of $v=\pi / 2$ is $0.308 \mu \mathrm{m}$ (the refractive index of the emulsion was approximately 1.62). The value of the thickness was $\sim 7 \mu \mathrm{m}$ for BB-640 emulsion, which permitted the highest value of diffraction efficiency to be achieved with a refractive-index modulation of $\Delta n \sim 0.044$. We show that rehalogenating bleaching techniques that do not include a fixation step are efficient enough to create this refractive-index modulation.

We tested the holograms by rotating them, and the variation in transmission with the angle of incidence $\tau$ in air was measured. The values of transmission were corrected to take into account Fresnel's reflections and the absorption of the glass substrate. We found the values of refractive-index modulation $\Delta n$, absorption coefficient $\alpha$, and thickness $d$ by fitting the theoretical function to the experimental data.

\section{Results and Discussion}

\section{A. Silver Halide-Sensitized Gelatin Holograms}

First we shall discuss the results obtained when SHSG holograms were recorded on BB-640 plates. Figure 2 shows the diffraction efficiency and the product $\Delta n d$ versus exposure for SHSG transmission holograms recorded on BB-640 plates. The values of the diffraction efficiency were measured, whereas the values of $\Delta n d$ were obtained by fitting of the theoretical response of the transmittance as a function of the reconstruction angle to the experimental data. The product $\Delta n d$ was calculated in the range of exposures in which Kogelnik's model is applicable ${ }^{15}$ (at high exposures, because of nonlinearities, the model is not completely valid). It can be seen that the curve of diffraction efficiency corresponds to typical behavior of diffraction efficiency versus exposure. The diffraction efficiency increases with exposure until a

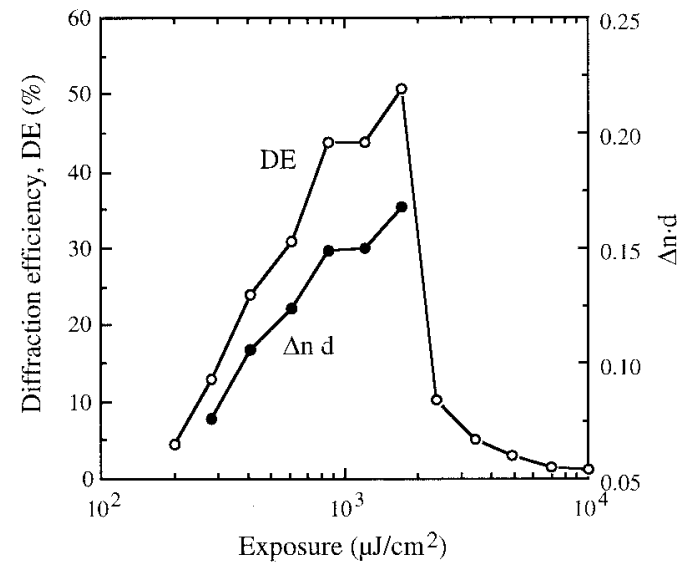

Fig. 2. Diffraction efficiency (DE) and $\Delta n d$ versus exposure for transmission SHSG holograms recorded on BB-640 plates at the Bragg angle. 

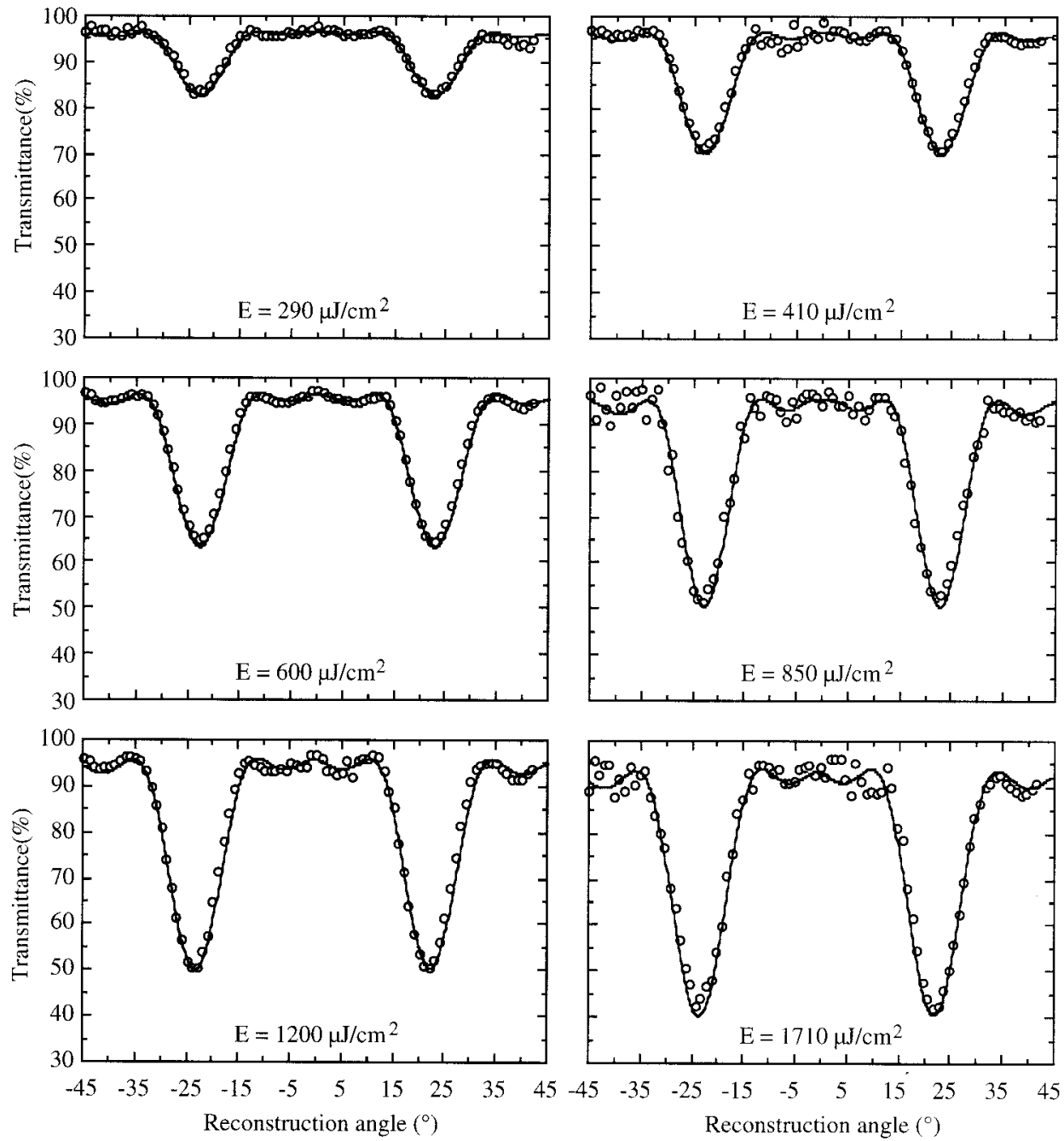

Fig. 3. Experimental measurements (circles) and theoretical fit (solid curves) of transmittance as a function of the reconstruction angle for SHSG transmission holograms recorded on BB-640 plates.

peak diffraction efficiency of $51 \%$ is reached at an exposure of $1700 \mu \mathrm{J} / \mathrm{cm}^{2}$. Then the diffraction efficiency decreases. In the gelatin there are no silver halide grains, so the refractive-index modulation must be due only to the differential degree of hardening of the gelatin, which occurs as a result of cross linking of the gelatin produced by the $\mathrm{Cr}^{+3}$ ion. ${ }^{16-20}$ The refractiveindex modulations obtained in this case are not so high as those obtained by rehalogenating bleaching, as we shall see in Subsection 4.B. The product $\Delta n d$ is kept below $0.308 \mu \mathrm{m}$ for the whole range of exposures, so the maximum diffraction efficiency is never achieved with this SHSG technique in this case.

Figure 3 shows transmittance versus angle at reconstruction for some SHSG phase transmission diffraction gratings recorded on BB-640 plates (those that correspond to the calculated values of $\Delta n d$ in Fig. 2). The theoretical fit corresponds to the continuous curves, whereas the open circles denote the experimental data. Theoretical curves were obtained with Eq. (4), as mentioned. Table 3 lists the values of $d, \Delta n$, and $\alpha d$ obtained by the theoretical fit for the six exposures analyzed. Typical behavior of transmittance as a function of the angle can be seen. For all the curves the transmission efficiency has a minimum at the Bragg angle that corresponds to a maximum diffraction efficiency at this angle. In addition, the troughs centered at the Bragg angle are deeper as the exposure increases, corresponding to an increase in diffraction efficiency. Good agreement between theory and experimental data can also be seen.

Table 3. Parameters $d, \Delta \mathrm{n}$, and $\alpha d$ for SHSG Transmission Gratings Recorded on BB-640 Emulsion

\begin{tabular}{cccc}
\hline$E\left(\mu \mathrm{J} / \mathrm{cm}^{2}\right)$ & $d(\mu \mathrm{m})$ & $\Delta n$ & $\alpha d$ \\
\hline 290 & 7.1 & 0.011 & 0.034 \\
410 & 6.9 & 0.015 & 0.034 \\
600 & 6.6 & 0.019 & 0.038 \\
850 & 7.1 & 0.021 & 0.048 \\
1200 & 6.5 & 0.018 & 0.043 \\
1710 & 6.3 & 0.027 & 0.062 \\
\hline
\end{tabular}




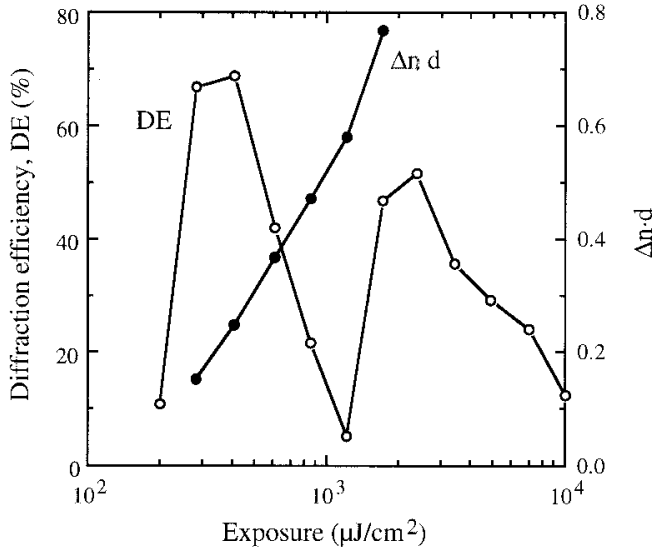

Fig. 4. Diffraction efficiency (DE) and $\Delta$ nd versus exposure for transmission bleached holograms recorded on BB-640 plates at the Bragg angle.

\section{B. Fixation-Free Rehalogenated Bleached Holograms}

Figure 4 shows diffraction efficiency and $\Delta n d$ versus exposure for bleached holograms recorded on BB-640 plates. It can be seen that diffraction efficiency increases with exposure until a value of $69 \%$ is reached for an exposure value of $400 \mu \mathrm{J} / \mathrm{cm}^{2}$; then the diffraction efficiency decreases to $5 \%$ at an exposure of $1200 \mu \mathrm{J} / \mathrm{cm}^{2}$, increases again to $52 \%$ at an exposure of $2400 \mu \mathrm{J} / \mathrm{cm}^{2}$, and then decreases again at very high exposures. This is in fact a different behavior from that commonly observed with Agfa plates and more closely resembles the behavior exhibited by the diffraction efficiency for SHSG diffraction gratings recorded on BB-640 plates analyzed in Subsection 4.A. It can be seen from the figure that the decrease in diffraction efficiency in the range of exposures from 400 to $1200 \mu \mathrm{J} / \mathrm{cm}^{2}$ is due to the high values of the product $\Delta n d$. This product surpasses the value of $0.308 \mu \mathrm{m}$, which corresponds to the maximum achievable diffraction efficiency in this range of exposures.

Figure 5 shows the angular dependence of the transmission efficiency for the phase transmission bleached holograms recorded on BB-640 plates at several values of exposure. Table 4 shows the re-
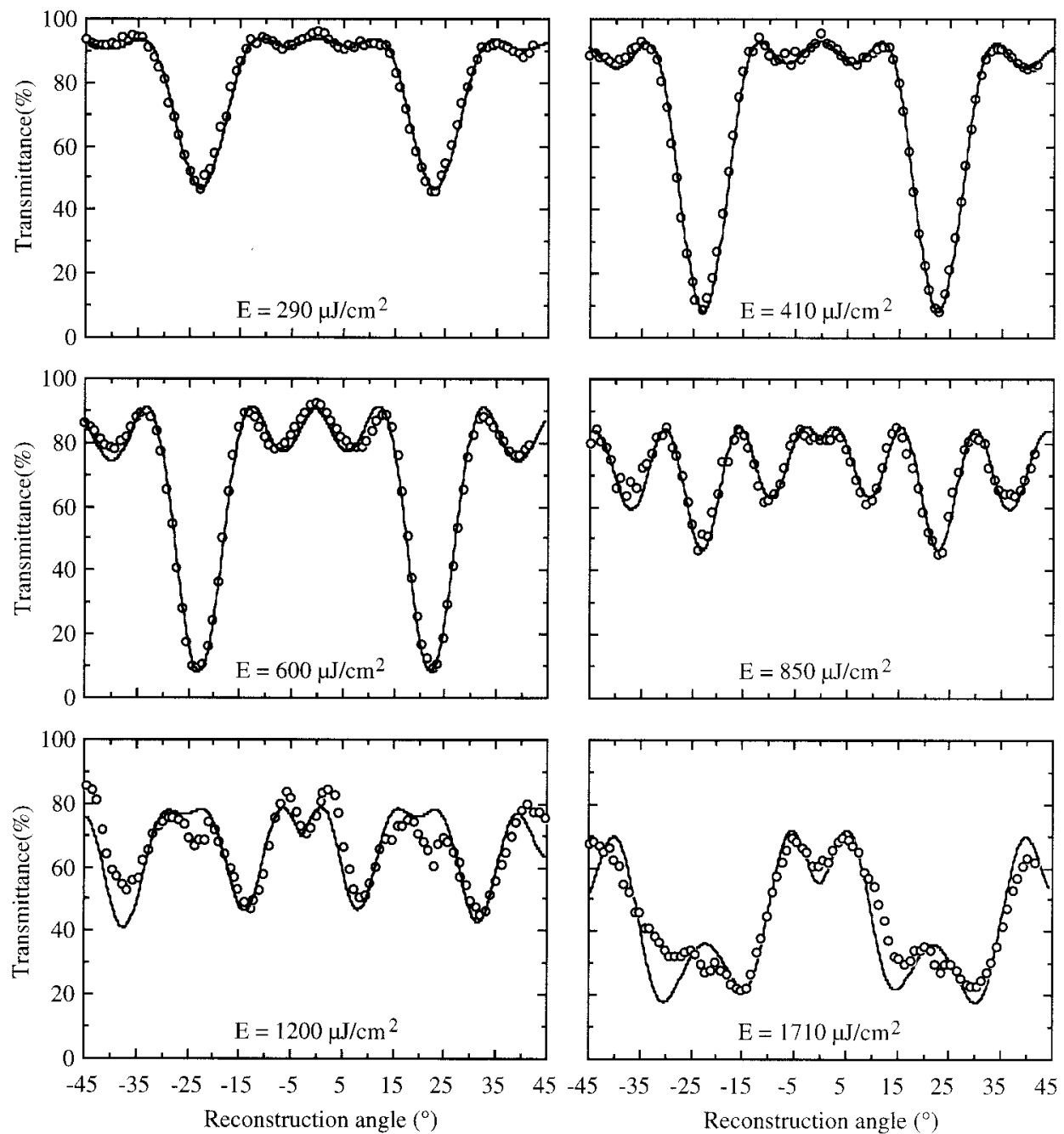

Fig. 5. Experimental measurements (circles) and theoretical fit (solid curves) of transmittance as a function of the reconstruction angle for fixation-free bleached transmission holograms recorded on BB-640 plates. 
Table 4. Parameters $d, \Delta \mathrm{n}$, and $\alpha d$ for Fixation-Free Rehalogenating Bleached Transmission Gratings Recorded on BB-640 Emulsion

\begin{tabular}{cccc}
\hline$E\left(\mu \mathrm{J} / \mathrm{cm}^{2}\right)$ & $d(\mu \mathrm{m})$ & $\Delta n$ & $\alpha d$ \\
\hline 290 & 6.7 & 0.023 & 0.062 \\
410 & 6.7 & 0.037 & 0.073 \\
600 & 6.3 & 0.058 & 0.089 \\
850 & 7.0 & 0.067 & 0.159 \\
1200 & 7.1 & 0.082 & 0.245 \\
1710 & 7.1 & 0.108 & 0.337 \\
\hline
\end{tabular}

sults of $d, \Delta n$, and $\alpha d$ in Fig. 5. For the first three values of exposure $\left(290,410\right.$, and $\left.600 \mu \mathrm{J} / \mathrm{cm}^{2}\right)$ the increase in exposure corresponds to an increase in the depth of the troughs centered at the Bragg angle, as was seen in Subsection 4.A for the SHSG holograms. Nevertheless, at exposures of 850, 1200, and 1700 $\mu \mathrm{J} / \mathrm{cm}^{2}$ the effective refractive-index modulations are high. The effect of these high modulations is to decrease the maximum achievable diffraction efficiency at the Bragg angle (depth of the troughs centered at the Bragg angle) and to increase the depth of the adjacent lobules. The immediate consequence of this behavior is that in the case of fixation-free rehalogenating techniques much care must be taken to control the excessive refractive-index modulation. The diffusion mechanism is efficient enough to create the necessary refractive-index modulation, and the usual methods of increasing this modulation (such as washing the final bleached holograms in subsequent baths of isopropanol) are not necessary; in fact, they could have a negative effect on the achievement of high diffraction efficiencies. A possible method for controlling excessive refractive-index modulation could be the use of hardener developers. The differential hardening of the gelatin created by the oxidation products of these developers acts against the diffusion mechanism, as explained by Hariharan. ${ }^{5}$ Therefore the refractive-index modulation could be limited by use of hardener developers that contain cathecol or pyrogallol or by reducing the amount of sodium sulfite in the D-19 developer, which would enhance the hardening effects of this developer. ${ }^{21}$

\section{Comparison of Rehalogenating Bleaching and SHSG Procedures}

Figure 6 shows refractive-index modulation versus exposure for phase holograms recorded on BB-640 plates: fixation-free rehalogenating bleached holograms and SHSG holograms. The theoretical function used to fit the experimental data was

$$
\Delta n(E)=\Delta n_{0}[1-\exp (-\beta E)]
$$

with the following fitted values of $\Delta n_{0}$ and $\beta$ :

$$
\Delta n_{0}=0.027 \text { and } \beta=0.00196 \mathrm{~cm}^{2} / \mu \mathrm{J} \text { for SHSG }
$$
holograms.

$\Delta n_{0}=0.160$ and $\beta=0.00064 \mathrm{~cm}^{2} / \mu \mathrm{J}$ for fixationfree rehalogenating holograms.

The value of $\Delta n_{0}$ is indicative of the maximum achievable refractive-index modulation (saturation modula-

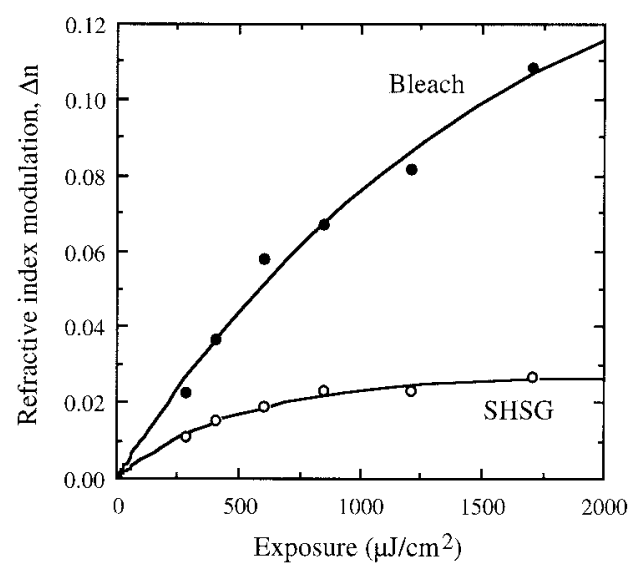

Fig. 6. Refractive-index modulation versus exposure for phase transmission holograms recorded on BB-640 plates.

tion). From the calculated data it can be seen that this value is considerably higher for bleached holograms. Furthermore, if the thickness of the final SHSG holograms is assumed to be $\sim 7 \mu \mathrm{m}$, the maximum achievable $\Delta n d$ can be only 0.189 , and so the maximum achievable diffraction efficiency cannot be reached with this SHSG method. By use of fixationfree rehalogenating techniques, the maximum achievable diffraction efficiency is clearly reached and even surpassed.

It has been demonstrated that the transfer of material is more efficient in creating refractive-index modulation than is differential hardening of the gelatin produced by the $\mathrm{Cr}^{+3}$ ion.

\section{Conclusions}

The mechanism for hologram formation in the case of fixation-free rehalogenating of bleached holograms (with potassium dichromate as the oxidizer) inside a BB-640 emulsion has been analyzed. It has been shown that high refractive-index modulations are created inside the emulsion and that these effectively lead to a decrease in diffraction efficiency at certain exposures. The results were compared with those obtained for the same plates and processed with SHSG. We have demonstrated that the $\mathrm{Cr}^{+3}$ ion is not so efficient in creating the high index modulations obtained by bleaching techniques. The results presented demonstrate that high diffraction efficiencies can be obtained by use of fixation-free rehalogenating techniques with transmission holograms recorded on BB-640 plates and that no additional methods are needed to increase the maximum diffraction efficiency. In fact, the aim of fixation-free bleaching techniques should be to control these high index modulations.

This study was supported by the Comision Interministerial de Ciencia y Tecnología of Spain as part of project MAT97-0705-C02-02.

\section{References}

1. L. Solymar and D. J. Cooke, Volume Holography and Volume Gratings (Academic, London, 1981), pp. 315-326. 
2. H. T. Buschman, "Bleichprozesse zur Erzeugung rauscharmer, lichtstarker Phasenhologramme," Optik (Stuttgart) 34, 240253 (1971).

3. P. Hariharan, C. S. Ramanathan, and G. S. Kaushik, "Simplified processing technique for photographic phase holograms," Opt. Commun. 3, 246-247 (1971).

4. J. Crespo, A. Fimia, and J. A. Quintana, "Fixation-free methods in bleached reflection holography," Appl. Opt. 25, 16421645 (1986).

5. P. Hariharan and C. M. Chidley, "Rehalogenating bleaches for photographic phase holograms: the influence of halide type and concentration on diffraction efficiency and scattering," Appl. Opt. 26, 3895-3898 (1987).

6. R. K. Kostuk, "Factorial optimization of bleach constituents for silver halide holograms," Appl. Opt. 30, 1611-1616 (1991).

7. P. Hariharan, "Basic processes involved in the production of bleached holograms," J. Photograph. Sci. Eng. 38, 76-81 (1990).

8. P. Hariharan, "Rehalogenating bleaches for photographic phase holograms. 3. Mechanism of material transfer," Appl. Opt. 29, 2983-2985 (1990).

9. R. Birenheide, "BB emulsions series: current standings and future developments," in Sixth International Symposium on Display Holography, T. H. Jeong, ed., Proc. SPIE 3358, 28-30 (1997).

10. A. Beléndez, C. Neipp, M. Flores, and I. Pascual, "Highefficiency silver-halide sensitized gelatin holograms with low absorption and scatter," J. Mod. Opt. 45, 1985-1992 (1998).

11. C. Neipp, I. Pascual, and A. Beléndez, "Silver halide sensitized gelatin derived from BB-640 holographic emulsion," Appl. Opt. 38, 1348-1356 (1999).
12. C. Neipp, I. Pascual, and A. Beléndez, "Optimization of a fixation-free rehalogenating bleach for BB-640 holographic emulsion," J. Mod. Opt. 47, 1671-1679 (2000).

13. A. Beléndez, C. Neipp, and I. Pascual, "Analysis and elimination of boundary reflections in transmission holograms," Opt. Laser Technol. 30, 555-556 (1999).

14. H. Kogelnik, "Coupled wave theory for thick hologram gratings," Bell Syst. Tech. J. 48, 2909-2947 (1969).

15. R. R. A. Syms, Practical Volume Holography (Clarendon, Oxford, UK, 1990), pp. 54-64.

16. W. R. Graver, J. W. Gladden, and J. W. Eastes, "Phase holograms formed by silver halide (sensitized) gelatin processing," Appl. Opt. 19, 1529-1536 (1980).

17. K. Biedermann, "Silver halide photographic materials," in $\mathrm{Ho}$ lographic Recording Materials, H. M. Smith, ed. (SpringerVerlag, Berlin, 1977), pp. 21-74.

18. D. Meyerhofer, "Dichromated gelatin," in Holographic Recording Materials, H. M. Smith, ed. (Springer-Verlag, Berlin, 1977), pp. 75-100.

19. G. Manivannan, R. Changkakoti, R. A. Lessard, G. Mailhot, and M. Bolte, "Primary photoprocesses of $\mathrm{Cr}(\mathrm{IV})$ in real-time holographic recording material: dichromated poly(vinyl alcohol)," J. Phys. Chem. 97, 7228-7233 (1993).

20. H. I. Bjelkhagen, Silver Halide Recording Materials for Holography and Their Processing (Springer-Verlag, Berlin, 1993), pp. 182-197.

21. P. Hariharan and C. M. Chidley, "Photographic phase holograms: the influence of developer composition on diffraction efficiency and scattering," Appl. Opt. 26, 1230-1234 (1987). 